\title{
Nanoelectrochemical Immunosensors for Protein Detection
}

\author{
Alessandro Carpentiero ${ }^{1}$, Manuela De Leo $^{2}$, Ivan Garcia Romero ${ }^{3}$, \\ Stefano Pozzi Mucelli ${ }^{4}$, Freimut Reuther ${ }^{3}$, Giorgio Stanta ${ }^{4}$, Massimo Tormen ${ }^{1, *}$, \\ Paolo $\mathrm{Ugo}^{2}$, and Martina Zamuner ${ }^{2}$ \\ ${ }^{1}$ Laboratorio Nazionale TASC-INFM-CNR, S.S. 14, Km. 163.5, I-34012 Trieste, Italy \\ ${ }^{2}$ Department of Physical Chemistry, University of Venice, Santa Marta 2137, \\ 30123 Venice, Italy \\ ${ }^{3}$ Micro Resist Technology GmbH, Koepenicker Str. 325, Haus 211, 1255 Berlin, Germany \\ ${ }^{4}$ Department of Clinical, Morphological and Technological Sciences, University of Trieste, \\ Strada di Fiume 447, 34149 Trieste, Italy and Molecular Histopathology Laboratory, \\ International Centre for Genetic Engineering and Biotechnology, Trieste, Italy
}

\begin{abstract}
Nanoelectrochemical immunosensors fabricated by templated electrodeposition of gold nanoelectrodes inside the pores of polycarbonate (PC) track-etched membranes, followed by the immobilization of the biorecognition elements on the surrounding PC, have proven high sensitivity and specificity for protein detection. The signal transduction scheme involves a suitable redox mediator added to the sample solution to shuttle electrons from the gold nanoelectrodes to the biorecognition layer, both elements being in strict spatial proximity. Highly improved signal-to-background current ratio, which are peculiar of NEEs with respect to other electrochemical transducers, can be exploited in this way. Two detection schemes were tested: one based on the direct immobilization of the target protein on the PC of the NEE (approach A) and the other based on the immobilisation on PC of an antibody to capture the target protein (approach B). The biorecognition process was completed by adding a primary antibody and a secondary antibody with horse radish peroxidase (HRP) as enzyme label; methylene blue was the redox mediator added to the electrolyte solution. Typical target analytes were single chain fragment variable proteins, for approach A, and trastuzumab (also known as Herceptin ${ }^{\circledR}$ ), for approach B. NEE-based capture sensors were tested successfully to detect small amounts of the receptor protein HER 2 in biological samples. Finally, motivated by the target of a better control of the geometrical characteristics of ensembles of nanoelectrodes (size, density, geometrical arrangement, and degree of recession), and by the positive results obtained with track-etch membranes of PC from the standpoint of protein immobilization, we demonstrated the fabrication of nanobiosensors by patterning ordered arrays of nanoelectrodes (NEAs) by electron beam lithography (EBL) on polycarbonate. EBL results perfectly suitable for the top-down fabrication of arrays of nanobiosensors on thin PC films deposited on gold coated silicon.
\end{abstract}

Keywords: Nanoelectrode, electrochemical immunosensor, proteomics, trastuzumab, voltammetry, electron beam lithography.

Corresponding author. 


\section{Introduction}

In electrochemical biosensors, a change in the redox state of the biorecognition/analyte system related to the biorecognition event, produces a change in an electrochemical quantity which can be monitored by classical electroanalytical techniques. Electrochemical systems can be miniaturized below the micrometer scale, using individual nanoelectrodes or ordered arrays or random ensembles of nanoelectrodes (NEA and NEE, respectively) [2]. In particular, the use of NEAs and NEEs allows to overcome problems related to the requirement for high signal amplification and careful shielding of electrical noise. Signals given by NEAs and NEEs are indeed the summation (weighed by diffusion effects) of the signals generated at each single nanoelectrode element [3]; a drawback is that NEAs and NEEs do not allow the extreme "nano-turization" degree achievable with individual nanoelectrodes, however they can be brought down to arrays of overall micro-size, but made of nanometer sized single components [4].

Among the others, NEEs prepared by controlled deposition of metal nanoelements using polycarbonate ultrafiltration membranes as template, are attractive and increasingly used, since their fabrication procedure is easy and accessible [5]. Electroless deposition of gold in polycarbonate templates for producing NEEs was introduced some years ago by Menon and Martin [3] and refined more recently in our laboratories [6]. Membranes with pore diameter from $10 \mathrm{~nm}$ to $10 \mu \mathrm{m}$ are commercially available. The diameter of the pores in the template determines the diameter of the Au-nanodisk elements that make up the NEE while the density of the pores in the membrane determines the number of $\mathrm{Au}$ - disk nanoelectrodes per $\mathrm{cm}^{2}$ and their average distance. NEEs can exhibit distinct voltammetric response regimes depending on the scan rate or distance between the nanoelectrode elements. [3] The total overlap (TO) regime is operative when radial diffusion boundary layers at each nanoelectrode overlap (slow scan rates and/or small distance between nanoelectrodes); this is the diffusion regime commonly observed for NEEs fabricated from commercial tracketched membranes. Under these conditions, the signal obtained at a NEE (namely, the faradaic peak current) is proportional to the geometric area $\left(\mathrm{A}_{\text {geom }}\right.$; that is area of the nanodisc electrodes plus insulating membrane between them), while the background current (i.e. double layer charging current) depends on the active area $\left(\mathrm{A}_{\text {act }}\right.$; area of the metal nanoelectrode elements alone) [4]. As a consequence of the high $\mathrm{A}_{\text {geom }} / \mathrm{A}_{\mathrm{act}}$ ratio, NEEs are characterized by detection limits 2-3 orders of magnitude lower than those achievable with regular electrodes of the same geometric area [7,8]. Some bioanalytical applications of arrays and ensembles of nanoelectrodes have been very recently reviewed [2].

In typical schemes used to obtain electrochemical biosensors, a biorecognition layer is directly immobilized on the electrode surface and the signal is produced by exchange of electrons between this layer and the underlying electrode. However, in the case of miniaturized electrodes, such as in the case of nanoelectrodes, the amount of immobilized biomolecules can be too small to give signals exploitable for analytical purposes. In order to increase the electrode area available for the immobilization, the template membrane can be etched [9-11]. However, this causes the loss of improved signal-to-background current ratios[11]. 
In a recent paper [12] we demonstrated the feasibility and the advantages of a different approach, that is, the immobilization of antibodies on the polycarbonate surrounding the nanodisk electrodes and not on the nanoelectrodes themselves. In such a design, transducer and biorecogniton layer are not overlapped, but integrated in strict proximity; since no increase in the $A_{\text {act }}$ is required, voltammetric signals are produced at the highest signal/background current ratio. The same scheme for the immobilization of the biorecognition element can in principle be extended to the case of NEA's, fabricated by highly controlled top-down lithographic processes, such as electron beam lithography (EBL). In fact, high resolution lithography allows a precise positioning of the nanoelectrodes as well as the control of their size down to the $10 \mathrm{~nm}$ scale. This is expected to improve even further signal/background ratios, to lower detection limits and to simplify the electrochemical data analysis, thanks to moving from randomly distributed nanoelectrodes in total overlap diffusion conditions to ordered arrays of electrochemical nanoelectrodes in non-overlapping diffusion conditions.

In the present study we report on i) the use of nanoimmunosensors based on tracketched membranes to determine two target proteins and on ii) the development of NEA's on PC by EBL as elements of the same strategy to reach nanobiosensors with superior performances.

Concerning the point i) the detection scheme was implemented for the single chain fragment variable $(\mathrm{ScFV})$ protein chosen as a general model protein, and on the HER2 receptor which represents a quite important target protein connected to the development of the so-called personalized therapies. The possibility to detect HER2 is indeed extremely important for the identification of cancer that can be treated successfully with Herceptin (Trastuzumab), a recombinant DNA-derived humanized murine monoclonal antibody directed against the extracellular domain of the HER2 receptor.

Relatively to point ii), we report on the advances in nanobiosensors fabricated by EBL on thin polycarbonate films on gold coated substrates, and their electrochemical characterization. Ordered nanoelectrodes were fabricated by patterning a thin $(\sim 100 \mathrm{~nm})$ PC film spin coated from solution on gold evaporated on silicon substrates. PC is increasingly used in biology as substrate for protein immobilization [13] and in electrochemistry [14]. However, at the best of our knowledge, it was never used before as resist for EBL. We present here the successful fabrication scheme of high resolution nanoelectrode arrays (NEAs) by EBL on PC and their preliminary characterization by cyclic voltammetry.

\section{Experimental Section}

\subsection{Apparatus and Materials}

All electroanalytical measurements were carried out at room temperature $\left(22 \pm 1^{\circ} \mathrm{C}\right)$ under a nitrogen atmosphere, using a three-electrode single-compartment cell equipped with a platinum coil counter electrode and an $\mathrm{Ag} / \mathrm{AgCl}$ ( $\mathrm{KCl}$ saturated) reference electrode. All potential values are referred to this reference electrode. A CH660A potentiostat controlled via PC by its own software was used for voltammetric measurements. 
All the used chemicals were reagent grade and utilized without further purification. Horseradish peroxidase, type VI, 298 Purpurogallin units/mg solid, was purchased from Sigma. Purified water was obtained using a Milli-Ro plus Milli-Q (Millipore) water purification system.

\subsection{Template Fabrication of NEEs}

NEEs were prepared by template gold electroless deposition [14], following recently described updates in the procedure[7,14,15]. The templating membranes were polycarbonate track-etched filter membranes (SPI-pore, $47 \mathrm{~mm}$ filter diameter, $6 \mu \mathrm{m}$ thickness) with a nominal pore diameter of $30 \mathrm{~nm}$, average pore density $6 \times 10^{8}$ pore $/ \mathrm{cm}^{2}$ and coated by the producer with the wetting agent polyvinylpyrrolidone.

Briefly, after wetting for $2 \mathrm{~h}$ in methanol, the polycarbonate template membrane was sensitized with $\mathrm{Sn}^{2+}$ by immersion into a solution that was $0.026 \mathrm{M}$ in $\mathrm{SnCl}_{2}$ and $0.07 \mathrm{M}$ in trifluoroacetic acid in 50:50 methanol-water for 5 minutes. After rinsing with methanol for $5 \mathrm{~min}$, the sensitized membrane was immersed for $10 \mathrm{~min}$ in 0.029 $\mathrm{M} \mathrm{Ag}\left[\left(\mathrm{NH}_{3}\right)_{2}\right] \mathrm{NO}_{3}$. The membrane was then immersed into the Au plating bath which was $7.9 \times 10^{-3} \mathrm{M}$ in $\mathrm{Na}_{3} \mathrm{Au}\left(\mathrm{SO}_{3}\right)_{2}, 0.127 \mathrm{M}$ in $\mathrm{Na}_{2} \mathrm{SO}_{3}$. After waiting 30 minutes, 0.625 $\mathrm{M}$ formaldehyde was added to the plating bath. The temperature of the bath was 0-2 ${ }^{\circ} \mathrm{C}$. Electroless deposition was allowed to proceed for 15 hours, after which an additional 0.3 M formaldehyde was added. Deposition was continued for another 9 hours, after which the membrane was rinsed with water and immersed in $10 \% \mathrm{HNO}_{3}$ for 12 hours. The membrane was then rinsed again with water and dried. For obtaining handy NEEs [14], the golden membrane was suitably attached to a copper tape, the outer face of the membrane was peeled with scotch tape and everything, apart ahole,was insulated by a layer of plastics (Monokote film by Topflite, that is polyethylene terephtalate film with a polyethylene layer which acts as heat activated adhesive). The hole determined the $\mathrm{A}_{\text {geom }}$ value, typically $0.07 \mathrm{~cm}^{2}$. The value of $\mathrm{A}_{\text {act }}$, estimated by SEM analysis, was $0.001 \mathrm{~cm}^{2}$.

\subsection{Detection Schemes and Immobilization Procedures}

The two detection strategies summarized in Figure 1 were used. In both approaches A and $\mathrm{B}$, the immobilization exploits the fact that polycarbonate contains carbonyl groups which can react with amine groups [16] of antibobies. Single chain fragment variable $(\mathrm{ScFv})$ antibodies were cloned in the vector pDAN5, purified from DH5 $\alpha E$. coli cells using a nickel affinity resin and diluted in bicarbonate buffer [17]. A microvolume of $0.5 \mu \mathrm{l}$ of a $4 \mu \mathrm{M} \mathrm{ScFv}$ solution was spotted onto the NEE, then incubated for 4 hours at $4^{\circ} \mathrm{C}$.

Electrodes were then washed with PBST (Phosphate Buffer Saline, added with $0,2 \% \mathrm{v} / \mathrm{v}$ Tween 20), and incubated for 10 minutes in $\mathrm{NaBH}_{4}\left(3.3 \times 10^{-2} \mathrm{M}\right.$ in ethanol: $\mathrm{PBST}=1: 3$ ) to block remaining carbonylic groups [18].

Then the NEE was incubated with an anti-SV5 antibody (in house purified, diluted 1:3000 in Phosphate Buffer Saline added with milk powder and Tween20 (PBSTmilk), and finally with an anti-mouse-HRP secondary antibody (Dako Cytomation, diluted 1:10000 in PBST-milk). Trastuzumab functionalised NEEs (T-NEE) were 


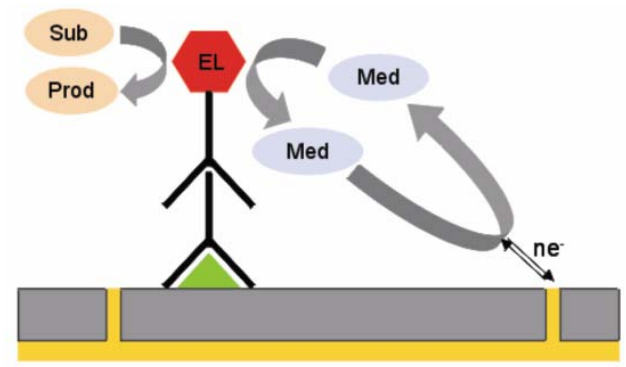

A)

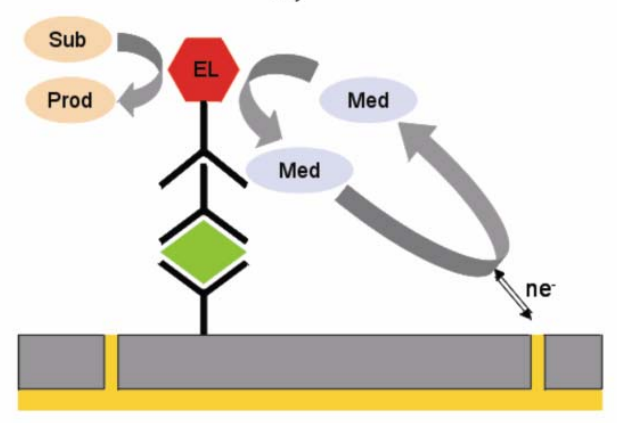

B)

Fig. 1. Schematic illustration of the two detection approaches used with NEEs: (A) the target protein is immobilized on the polycarbonate membrane, then it binds a primary antibody and secondary antibody with the enzyme label (EL); a soluble mediator (Med) shuttles electrons from the nanoelectrode to EL. (B) A primary antibody, specific for the target protein, is first immobilized on the membrane, in order to capture the target protein which is finally detected via primary plus secondary labelled antibody.

prepared by incubating on the NEE $2 \mu \mathrm{l}$ of $0.5 \mu \mathrm{g} / \mu \mathrm{l}$ trastuzumab (Herceptin ${ }^{\circledR}$, Genentech, South San Francisco, CA) in carbonate buffer, for 2 hours at $4^{\circ} \mathrm{C}$. After washing with PBST, $10 \mu \mathrm{l}$ of four dilutions $(1: 1,1: 5,1: 10,1: 50)$ of a $10 \mu \mathrm{g} / \mu \mathrm{l} \mathrm{SKBR} 3$ lysate were incubated with the T-NEE for 4 hours at $4^{\circ} \mathrm{C}$; the monoclonal antibody CB-11, diluted 1:5000 in PBST-milk, was used to detect bound Her2, together with an antimouse HRP-conjugated antibody.

NEEs used as negative control were not incubated with the cell lysate, but, right after the immobilization of trastuzumab they were incubated with the CB-11 antibody and the secondary antibody.

\subsection{Fabrication of NEAs by EBL}

A commercial Lexan polycarbonate sheet dissolved in dichloromethane is used as resist, spin coated to a thickness of 50 to $200 \mathrm{~nm}$ on an $\mathrm{Au} / \mathrm{Cr}(20 / 10 \mathrm{~nm})$ film evaporated on silicon, and pre-baked at $170^{\circ} \mathrm{C}$ for $5 \mathrm{~min}$. Test patterns were exposed to a focused electron beam in a Zeiss $1540 \mathrm{XB}$ Cross Beam system. Given the unavailability of literature data for optimal exposure dose of PC for electron beam lithography, we tested a broad dose range between $20 \mu \mathrm{C} / \mathrm{cm}^{2}$ to $20000 \mu \mathrm{C} / \mathrm{cm}^{2}$ at 30 
$\mathrm{kV}$ acceleration voltage. The development was done in $\mathrm{NaOH} 5 \mathrm{M}$ aqueous solution at different temperatures between 40 an $70{ }^{\circ} \mathrm{C}$, followed by rinsing in water.

\section{Results and Discussion}

\subsection{Tests of NEEs as Nanobiosensors}

Preliminary tests performed in homogeneous solutions (mediator, enzyme and substrate all dissolved in solution), showed that the best mediator suitable to shuttle electron from the Au-nanoelectrodes of a NEE to HRP was methylene blue (MB) which is reduced reversibly at the NEE according to the following reaction[12]:

$$
\mathrm{MB}+2 \mathrm{e}+\mathrm{H}^{+} \rightarrow \mathrm{LB}
$$

where LB is the leuco (reduced) form of MB.

In the presence of $\mathrm{HRP}$ and its substrate $\mathrm{H}_{2} \mathrm{O}_{2}, \mathrm{MB}$ shuttles electrons from the nanoelectrode to the enzyme label being involved in the following electrocatalytic cycle:

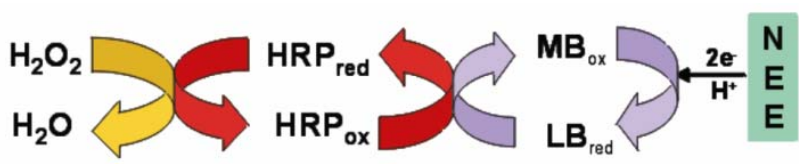

In order to test the efficiency of the detection scheme shown in Fig.1A, further tests were performed by immobilizing $\mathrm{ScFv}$ on the NEE surface. The protein was then bound to anti-SV5 antibody plus anti-mouse-HRP secondary antibody. The tests were performed by adding increasing concentration of $\mathrm{H}_{2} \mathrm{O}_{2}$, thus allowing one to determine also the best operative range for the concentration of the enzyme substrate. As shown in Figure 2, even at relatively low concentration of $\mathrm{H}_{2} \mathrm{O}_{2}$ (namely, $1.5 \mathrm{mM}$ ) the voltammetric patterns assumed the typical sigmoidal electrocatalytic shape (full line). These results confirmed the feasibility at NEE of the detection scheme $1 \mathrm{~A}$, at least when using protein samples (like the $\mathrm{ScFv}$ sample tested here) very rich in analyte. Methylene blue signals (see broken line in Fig. 2) at the NEE functionalised with ScFv- anti-SV5-anti-mouse-HRP (see broken line in Fig. 3) did not show significant differences with the signal recorded at unmodified NEEs, so indicating no inhibition by the immobilized proteins on the charge transfer at the surface of the gold nanoelectrodes. The above evidences support the feasibility of the detection scheme 1A, at least when high concentration of the target protein is present in the sample. However, to the goal of analysing with NEEs samples containing small concentration of the target protein, the development of capture arrays based on the scheme in Fig.1B appeared more suitable. To this aim, we tested as capture agent trastuzumab, which is a monoclonal humanized antibody used in the adjuvant therapy of breast cancer [19]. At first, preliminary immunoprecipitation assays were performed. As negative control, we immunoprecipitated our sample using an anti-PCNA (Proliferating cell nuclear antigen, Santa Cruz Biotechnology, CA) antibody, while as positive control, $70 \mu \mathrm{g}$ of whole SKBR3 [20] lysate were loaded onto the gel. Western blot analysis 


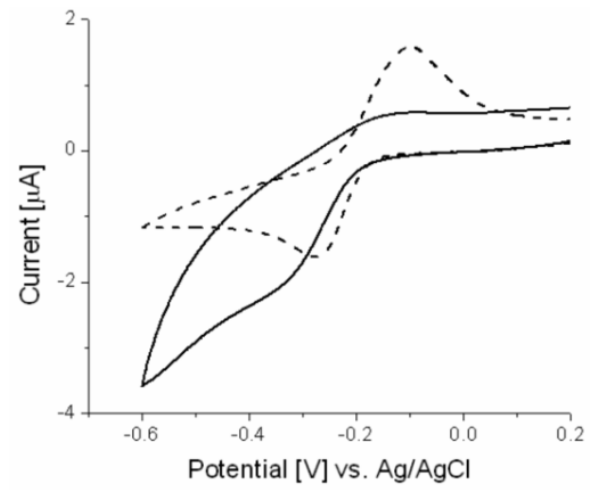

Fig. 2. Cyclic voltammograms recorded at a NEE functionalised with the target protein $\mathrm{ScFv}$, the primary anti-SV5 antibody and the anti-mouse-HRP secondary antibody; in solution containing $0.1 \mathrm{mM}$ methylene blue before (dash line) and after adding $1.5 \mathrm{mM} \mathrm{H}_{2} \mathrm{O}_{2}$ (full line). Scan rate: $50 \mathrm{mV} / \mathrm{s}$, supporting electrolyte $10 \mathrm{mM}$ phosphate buffer, $\mathrm{pH} 7$.

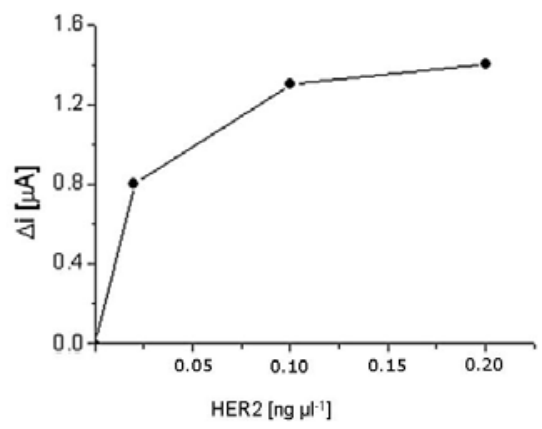

Fig. 3. Dependence of electrocatalytic current increase $(\Delta \mathrm{i})$ on HER 2 concentration at a NEE functionalized with trastuzumab. Cell lysates containing the target protein were diluted 1:10 (full line) and 1:50 (dashed line).

performed after the immunoprecipitation, indicated the bands, at $185 \mathrm{kDa}$ and $95 \mathrm{kDa}$, both due to the Her2 protein, were clearly visible, so confirming [21] the efficiency of trastuzumab as capture agent in vitro.

The tests were then performed with NEEs functionalized with Trastuzumab (see experimental section). The mediator, namely $0.1 \mathrm{mM} \mathrm{MB}$, and the substrate $(1.5 \mathrm{mM}$ $\mathrm{H}_{2} \mathrm{O}_{2}$ ) were added to the PBS electrolyte. The cyclic voltammetric patterns for the mediator MB at a NEE after the immobilization of trastuzumab, recorded in the absence and in the presence of cell lysate, but always without HER2, overlap each other. This confirms that no change in the voltammetric behaviour of MB at the NEE is caused by functionalization with trastuzumab. Moreover, no aspecific adsorption of the labeled antibody occurs if the target protein (HER2) is not present in the sample.

On the other hand, a significant increment in the current intensity $(\Delta \mathrm{I})$ was observed after incubation in the cell lysates, containing the target protein HER2, followed by incubation with the primary antibody and secondary antibody with the HRP 
label. Some tests were performed by changing the HER2 concentration in the sample by proper dilution of a cell lysate $(2 \mathrm{ng} / \mu \mathrm{l}$ before dilution) standardized by Western blot analysis, with a HER2 content of $1 \mu \mathrm{g} / \mu \mathrm{l}$, before dilution. The content of total proteins in the same undiluted sample was $10 \mu \mathrm{g} / \mu \mathrm{l}$. The immobilization of the all four-proteins chain (specific antibody, target protein, primary antibody and secondary labeled antibody) hinders slightly, but do not block the electron transfer. This suggests that the protein chain is immobilized in very close proximity to the gold nanodisks, but not directly on them. The voltammogram recorded in the presence of 1.5 $\mathrm{mM} \mathrm{H} \mathrm{H}_{2} \mathrm{O}_{2}$ shows a typical electrocatalytic character, with electrocatalytic $\Delta \mathrm{I}$ values which increase with the HER2 concentration as shown in Fig. 3. The concentration of $\mathrm{H}_{2} \mathrm{O}_{2}$ here used corresponds to the value which gives the highest electrocatlytic increase in peak currents, without causing an undesired increase in background current, as observed by us for instance when 3 or $5 \mathrm{mM} \mathrm{H}_{2} \mathrm{O}_{2}$ was added to the sample.

Note that the sensitivity of classical immunochemical assays, such as Western blotting, is not high enough to detect the HER2 analyte at the dilution levels of our 1:10 or 1:50 samples, while good electrocatalytic signals were detected with the NEE in these diluted samples. Preliminary tests on lysates of cancer cells overexpressing HER2 were successful and encouraging for the application of the NEE based biosensor to analyses of clinical samples [12].

\subsection{Lithographic Tests on NEAs Fabrication on PC and Preliminary Electrochemical Characterization}

In developing electron beam lithography on polycarbonate, we took inspiration from the fabrication technology of Track-etch Membranes (TM). In TM technology heavy ions, such as Xe accelerated to energies of several $\mathrm{MeV}$ or fragments of nuclear fission of ${ }^{235} \mathrm{U}$ are used to produce long straight cylindrical pores in polymer materials such as polyethylene terephthalate (PET) or polycarbonate (PC). Energetic heavy ions in passing in the material create tracks which etch in concentrated alkaline solutions $(\mathrm{NaOH})$ at a very different rate $\mathrm{V}_{\mathrm{t}}$ from that of bulk $\mathrm{V}_{\mathrm{b}}$. In polycarbonate etch rate ratio $\mathrm{V}_{\mathrm{t}} / \mathrm{V}_{\mathrm{b}}$ between $10^{2}$ and $10^{5}$ can be achieved by development in aqueous alkaline solutions [22,23]. In the present work we adapted the process used to produce PC track-etch membrane to the case of radiation damage produced by an energetic electron beam by controlled in a modern generation EBL system.

In our work the lithographic tests were made on $\mathrm{Au}$ film evaporated on silicon (with an intermediate Cr layer for adhesion). The motivation for that choice is twofold: 1) gold will act as recessed electrode after the opening of holes in the polycarbonate film in the final electrochemical nanosensors; 2) polycarbonate can not be considered as a normal resist. In fact, it could not be used directly, for instance, on silicon which would be etched during the development of polycarbonate in $\mathrm{NaOH}$ solution. Therefore the $\mathrm{Au} / \mathrm{Cr}$ film is necessary also from the point of view of the protection of silicon substrate during the lithographic process. The pattern for the dose matrix consisted of a $10 \times 10$ array of dot arrays exposed at $30 \mathrm{kV}$ acceleration voltage with doses from $20 \mu \mathrm{C} / \mathrm{cm}^{2}$ to $20000 \mu \mathrm{C} / \mathrm{cm}^{2}$. The development process conditions were also tested by varying the temperature of the $\mathrm{NAOH}$ aqueous solution from 30 to $70{ }^{\circ} \mathrm{C}$. 

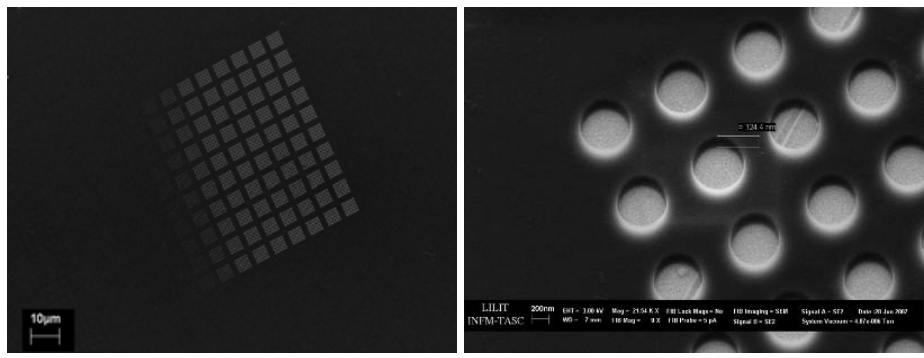

Fig. 4. Right. SEM image of 10x10 array of dots at different exposure dose. The first column is not visible due to the insufficient dose. Left. Detail of an array of dots at the exposure dose of $5000 \mu \mathrm{C} / \mathrm{cm}^{2}$.

When the development processes was carried out at $70^{\circ} \mathrm{C}$ we observed the formation of large pinholes in the polymer film, suggesting that the suitable temperature window for the development is in the range from RT and $60{ }^{\circ} \mathrm{C}$. In particular, we found that the optimal dose for development performed at $45^{\circ} \mathrm{C}$ is around $2000 \mu \mathrm{C} / \mathrm{cm}^{2} @ 30 \mathrm{KV}$.In a final test we tried to explore the resolution by decreasing the minimum feature size. In order to inspect high resolution pattern (holes), and to verify that the development had reached the substrate we found convenient to electroplate a layer of gold inside the holes using $\mathrm{Au} / \mathrm{Cr}(20 / 5) \mathrm{nm}$ film as base for the plating. High resolution dots electroplated inside holes and trenches in Lexan after removal of the polymer. Dots of size down to $50 \mathrm{~nm}$ were obtained, showing the suitability of PC for high resolution patterning by EBL.
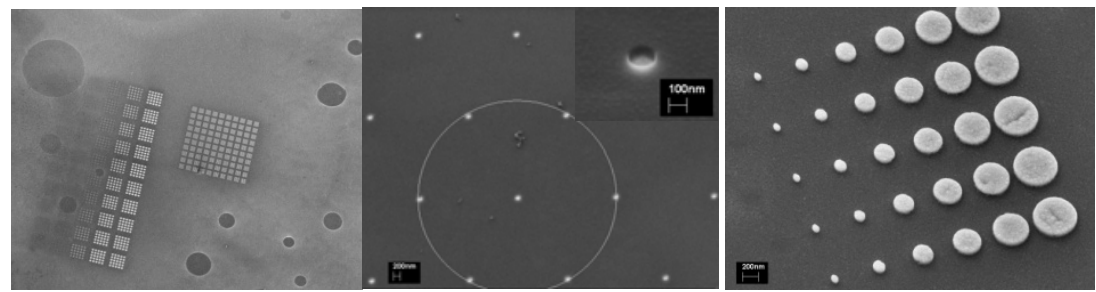

Fig. 5. Left: SEM micrograph of a dose matrix test developed at $70^{\circ} \mathrm{C}$ for $60^{\prime \prime}$. Large pinholes appear on the polycarbonate film. Center: top view of $75 \mathrm{~nm}$ radius dots in hexagonal array on PC film after E-beam exposure in order to obtain NEA. Right: electroplated gold dots of various size down to $50 \mathrm{~nm}$, proving that the holes were completely opened during development.

Fig. 6 shows the cyclic voltammograms recorded with a NEA (Fig. 5, center) in 10-4 M $\alpha$-methylferrocenemethanol (FE) solution, in aqueous solution of $\mathrm{NaNO}_{3}$ supporting electrolyte at two different concentrations, namely 0.01 or $0.5 \mathrm{M}$ (blue and red line, respectively). Both voltammetric patterns are sigmoidally shaped; this indicates the achievement of a radial diffusion regime, in which the diffusion hemisphere around the individual nanoelectrodes do not interfere (or overlap) each other $[8,13]$. These results show clearly that our polycarbonate NEA fabricated by EBL were fully functional and suitable for the development of electrochemical nanobiosensors. 


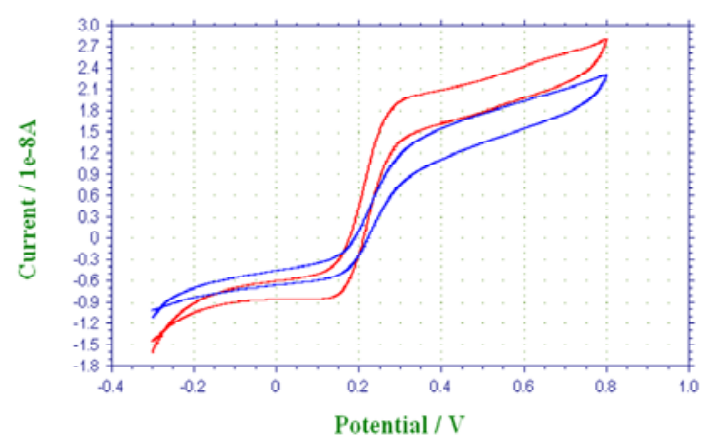

Fig. 6. CVs recorded with a NEA in $10^{-4} \mathrm{M}$ FE $0.5 \mathrm{M} \mathrm{NaNO}_{3}$ (red line) and $0.01 \mathrm{M} \mathrm{NaNO}_{3}$ (blue line) Scan rate : $10 \mathrm{mV} / \mathrm{s}$

\section{Conclusions}

The results presented here confirm the potentiality of electrochemical nanobiosensors based on ensembles of nanoelectrodes for the sensitive determination of target proteins, since with NEEs it was possible to detect target proteins in rather diluted samples (at least down to $20 \mathrm{pg} / / \mu \mathrm{l}$ ), where traditional, immunochemical method, such as Western blotting, failed for lack of sensitivity. Further studies aimed at validating the analytical performances of the sensor in terms of detection limit, dynamic range, accuracy and precision, are in progress.

Moreover, a new polycarbonate resist was tested as electron beam lithography resist, in a broad range of doses between $20 \mu \mathrm{C} / \mathrm{cm}^{2}$ to $20000 \mu \mathrm{C} / \mathrm{cm}^{2}$ at $30 \mathrm{kV}$ acceleration voltage, with different development process and temperatures, from RT to $70^{\circ} \mathrm{C}$. High resolution patterning of electrochemical nanosensors together with their correct functionality for cyclic voltammetry was demonstrated.

These two results together open the perspective of obtaining in a highly controlled manner electrochemical nanobiosensors on thin films of polycarbonate, a material chemically stable in common electrolytes and on which antibodies can be immobilized by using standard chemical functionalization procedures.

\section{Acknowledgements}

Financial support by MUR (Rome) is gratefully acknowledged.

\section{References}

1. Cunningham, A.J.: Introduction to Bioanalytical Sensors. J. Wiley \& Sons, New York (1998)

2. Shi, H.B., Yeh, J.I.: Nanomedicine 2, 587-598 (2007)

3. Menon, V.P., Martin, C.R.: Anal. Chem. 67, 1920-1928 (1995)

4. Moretto, L.M., Pepe, N., Ugo, P.: Talanta 62, 1055-1060 (2004) 
5. Ugo, P., Pepe, N., Moretto, L.M., Battagliarin, M.: J. Electroanal. Chem. 560, 51 (2003)

6. Pereira, F.C., Moretto, L.M., De Leo, M., Boldrin Zanoni, M.V., Ugo, P.: Anal. Chim. Acta 575, 16-24 (2006)

7. Ugo, P., Moretto, L.M., Bellomi, S., Menon, V.P., Martin, C.R.: Anal. Chem. 68, 4160-4165 (1996)

8. Ugo, P., Moretto, L.M., Vezzà, F.: ChemPhysChem 3, 917-925 (2002)

9. Yu, S., Li, N., Wharton, J., Martin, C.R.: Nano Lett. 3, 815-818 (2003)

10. De Leo, M., Kuhn, A., Ugo, P.: Electroanalysis 19, 227-236 (2007)

11. Lapierre-Devlin, M.A., Asher, C.L., Taft, B.J., Gasparac, R., Roberts, M.A., Kelley, S.O.: Nano Lett. 5, 1051-1055 (2005)

12. Pozzi Mucelli, S., Zamuner, M., Tormen, M., Stanta, G., Ugo, P.: Biosens. Bioelectron 22, 1900-1903 (2008)

13. Souplet, V., Desmet, R., Melnyk, O.: Bioconjugate Chem. 20, 550-557 (2009)

14. Ugo, P., Moretto, L.M.: In: Zoski, C.G. (ed.) Handbook of Electrochemistry, ch.16, Section 16.2, pp. 678-709. Elsevier, Amsterdam (2007)

15. De Leo, M., Pereira, F.C., Moretto, L.M., Scopece, P., Polizzi, S., Ugo, P.: Chem. Mater. 19, 5955-5964 (2007)

16. Rucker, V.C., Havenstrite, K.L., Simmons, B.A., Sickafoose, S.M., Herr, A.E., Shediac, R.: Langmuir 21, 7621-7625 (2005)

17. Sblattero, D., Bradbury, A.: Nat. Biotechnol. 18, 75-80 (2000)

18. Afanassiev, V., Hanemann, V., Wolfl, S.: Nucleic Acids Res. 15, e66 (2000)

19. Simonds, H.M., Miles, D.: Expert Opin Biol Ther. 7, 487-491 (2007)

20. Pasleau, F., Grooteclaes, M., Gol-Winkler, R.: Oncogene 8, 849-854 (1993)

21. Molina, M.A., Codony-Servat, J., Albanell, J., Rojo, F., Arribas, J., Baselga, J.: Cancer Res. 61, 4744-4749 (2001)

22. Guillot, Rondelez: J. Appl. Phys. 52, 7155-7164 (1981)

23. Spohr, R.: Radiation Measurements 40, 191-202 (2005) 